\title{
Accurate Airway Wall Estimation Using Phase Congruency $^{\star}$
}

\author{
Raúl San José Estépar ${ }^{1}$, George G. Washko ${ }^{2}$, Edwin K. Silverman ${ }^{2,3}$, \\ John J. Reilly ${ }^{2}$, Ron Kikinis ${ }^{1}$, and Carl-Fredrik Westin ${ }^{1}$ \\ 1 Surgical Planning Lab, Brigham and Women's Hospital, Boston, MA \\ \{rjosest, westin, kikinis\}@bwh.harvard.edu \\ ${ }^{2}$ Pulmonary and Critical Care Division, \\ Brigham and Women's Hospital, Boston, MA \\ \{gwashko, jreilly\}@partners.org \\ ${ }^{3}$ Channing Laboratory, Brigham and Women's Hospital, Boston, MA \\ reeks@channing. harvard.edu
}

\begin{abstract}
Quantitative analysis of computed tomographic (CT) images of the lungs is becoming increasingly useful in the medical and surgical management of subjects with Chronic Obstructive Pulmonary Disease (COPD). Current methods for the assessment of airway wall work well in idealized models of the airway. We propose a new method for airway wall detection based on phase congruency. This method does not rely on either a specific model of the airway or the point spread function of the scanner. Our results show that our method gives a better localization of the airway wall than "full width at a half max" and is less sensitive to different reconstruction kernels and radiation doses.
\end{abstract}

\section{Introduction}

Chronic obstructive pulmonary disease (COPD) is a general term used to describe a family of conditions that affects the lung tissue (emphysema) and airways. Pathologically, emphysema is the enlargement and permanent destruction of the air compartments distal to the terminal bronchiole and airways disease is characterized by lumenal obstruction due to wall inflammation, thickening and smooth muscle constriction. While both processes are associated with exposure to agents such as cigarette smoke, the susceptibility to development of COPD and the relative contributions of airway and airspace disease in an individual is highly variable. The development of precise and reproducible methods to accurately quantify the amount of airways disease and emphysema will facilitate efforts to understand the genetic and mechanistic pathways for disease development and may help optimize the selection of therapy for patients. Computed tomographic imaging of the lung has allowed researchers to examine the architecture of the diseased lung in-vivo. Recent investigations using quantitative image analysis of CT datasets have provided new insights into the characterization of

\footnotetext{
* This work has been funded by grants R01 HL075478, R01 HL68926 and P41RR13218.
} 
both emphysema and airways disease. Measurements of airway wall thickness on CT images are predictive of lung function; thicker airway walls tend to be found in subjects with reduced airflow 5 .

In this paper we have focused on the accurate estimation of the wall thickness. Such measurements requires detecting the inner interface between lumen and airway wall and the outer interface between airway wall and the surrounding lung tissue. While the definition of the inner part of the wall is characterized by a clear air-tissue interface, defined by an increase in the intensity profile, the outermost part of the wall poses greater difficulty. The airways are surrounded by parenchymal tissue and vessel structures.

The traditional approach to perform wall thickness detection has relied on the so-called "full-width at half-max" (FWHM) principle. This method defines the wall boundaries by considering the point with half intensity with respect to the peak intensity inside the wall. It is based on the fact that under an ideal step function that undergoes a Gaussian blurring, the edge is located in the FWHM location. Gaussian blurring is the de-facto model for the point spread function of a scanner. This method, although simple, has two major drawbacks:

- The airway wall can be considered as a laminated structure whose scale is close to the scanner scale resolution. This violates the perfect edge principle that FWHM is based on. The result is that, as reported by Reinhardt et al. 7, the measurements yields by FWHM are biased towards underestimation/overestimation of the inner/outer boundary respectively.

- The geometry of the wall is assumed to be circular. In this sense, the airway can be analyzed in polar coordinate system by just looking in the radial direction. Airways that do not fall in the orthogonal to the scanning direction do not fulfill this requirement.

Regarding the first problem, in [7] the authors propose a model-based fitting method that assumes a Gaussian point spread function and an idealized steplike model for the airway. After fitting the model to the data, the wall thickness is derived from the model. This method, although highly accurate, is very computationally intensive and does not take into account deviations from the proposed model that often happen; for example, areas where the airway wall is in contact with a vessel. Regarding the second problem, Sabba et al. 8 have proposed a method that assumes an elliptical model of the airway and a full 3D point spread function and does a fitting of the model to the actual image. The method improves the location of the lumenal edge; however the outer edge is not improved.

In this paper we propose a new method for wall thickness estimation that has as primary features of being model free, in terms of wall shape or blurring mechanism, and appears to be quite accurate. The method is based on the phase congruency principle for the location of edges. Under phase congruency, the location of relevant features, i.e. edges or lines, is given by those locations where the local phase exhibits maximal coherency. We will show that phase congruency is present at the scanner level when reconstructing the data with different reconstruction kernels. This has allowed us to lay the basis for a bronze 


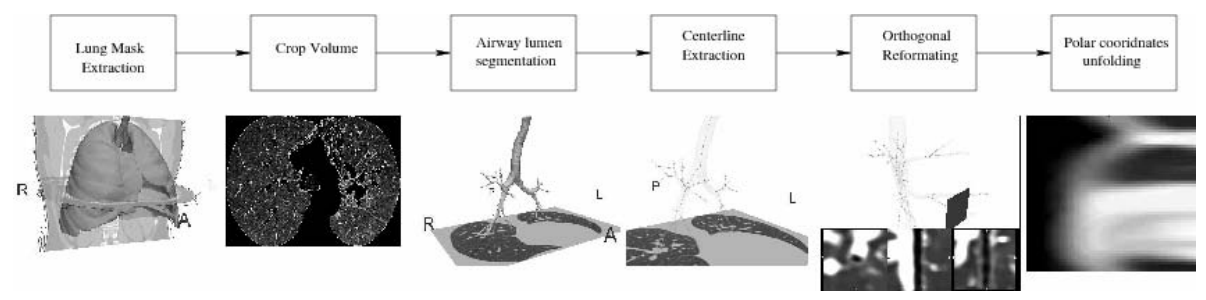

Fig. 1. Analysis pipeline for the extraction of unfolded orthogonal airways from lung CT datasets

standard for airway wall localization. We have characterized and validated using synthetic and real datasets.

\section{Airway Extraction Pipeline}

Before proceeding to the presentation of our method, we briefly describe the processing pipeline that we follow to extract unfolded airway volumes as depicted in Fig. 1. The lung mask is extracted by performing a thresholding at $-600 \mathrm{HU}$, applying connected components from a pre-estimated seed points falling in the left and right lung centroid and removing inside holes by means of mathematical morphology [2]. From the lung mask, the CT volume is cropped to the bounding box enclosing the lung and the intensity variations outside the lung are set to a fixed value. This cropped volume is used as an input to a surface evolution method to extract the airway lumen. The evolution is implemented by a fast marching approach initially proposed by Pichon et al. [6] for brain segmentation. The propagation is stopped when the physical volume of the segmented region sharply increases, being an indicator of leakage into the lung parenchyma. Once the airway lumen is segmented, the centerline is extracted by means of a thinning process 3 . From the centerline, the CT volume can be resampled in orthogonal planes to the centerline producing a flat airway volume. The flattened airway can be analyzed slice by slice for the task of airway wall detection. To simplify the analysis even further, the $2 \mathrm{D}$ flattened airway is unfolded in a polar coordinate system to work on $1 \mathrm{D}$ profiles cast from the airway center.

\section{Method}

\subsection{CT Reconstruction and Phase}

To provide a basis for the use of phase congruency, we present an experiment that reveals the variations associated with the use of different reconstruction kernels from the same CT acquisition. A CT image was reconstructed using a series of 9 different kernels and the same airway was selected and analyzed across the range of resulting images. Common intensity profiles projecting radially from the center of the airway were superimposed and displayed graphically (Fig. 2a). 


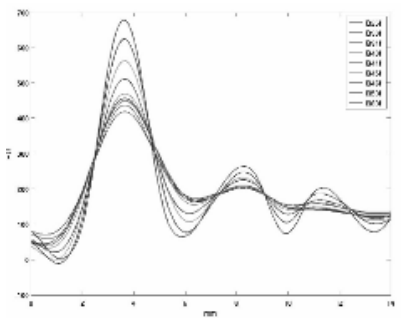

(a)

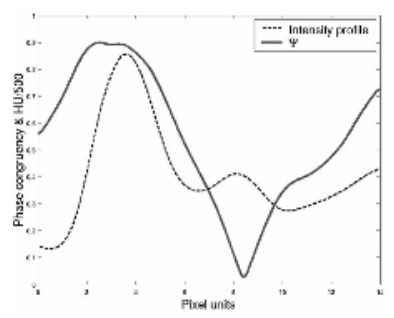

(b)

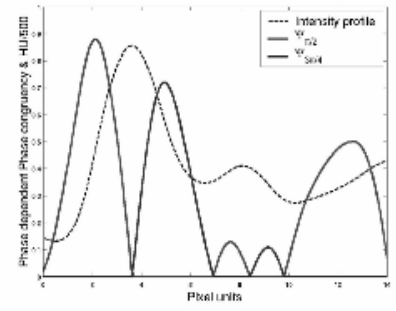

(c)

Fig. 2. (a) Intensity profiles along the radial direction of an airway for 9 different reconstruction kernels. Kernels B25f and B60f correspond to the smoothest and sharpest modulation transfer function respectively. (b)-(c) Comparison between the traditional phase congruency measurement (b) and the proposed phase congruency (c). The intensity profile that the phase congruency is estimated from is plotted in dash line.

On visual inspection, it can be seen that the peak intensity corresponding to the wall center is a function of the chosen kernel while the average intensity in the lumenal and parenchymal area are roughly constant. This implies that the FWHM method is going to yield a different result depending on the chosen reconstruction kernel.

An interesting point is revealed in Figure 2 2 . The profiles exhibit a common crossing point in the location of the inner and outer wall. This is replicated across the whole airway and across all airways assessed (data not shown). This crossing point is associated with the concept of phase congruency. Based on the model for feature perception postulated by Morrone and Owens [4, features on a image are perceived in those locations where the Fourier components of the local signal are maximally in phase. The features are associated with changes of intensity due to the presence, in our case, of an interface. This finding suggests that the crossing point of the intensity profile for different reconstruction kernels can be used as a bronze standard for airway wall location in real datasets. The results that we will present after this section corroborate this contention.

\subsection{Phase Congruency}

Local phase is the spatial counterpart of the widely known concept of Fourier phase. From a mathematical standpoint, the local phase is defined from the analytic signal. The analytic signal of a $1 \mathrm{D}$ real signal $f(x)$ is a complex value signal whose real part is the signal itself and the complex part is the Hilbert transform of the real signal

$$
f_{A}(x)=f(x)-i \mathcal{H}\{f(x)\}=E(x) e^{i \phi(x)} .
$$

$E(x)$ is the local energy of the signal and $\phi(x)$ is the local phase. Local phase is meaningful for signals that are well localized in the frequency domain. For a signal with a wide frequency spectrum, like any signal that can be found in real 
applications, a frequency localization has to be carried out before computing local phase. In this sense, it is possible to achieve different measurements of the local phase for different bands, i.e. different scales.

$$
f_{A_{n}}(x)=h_{n}(x) * f(x)+i \mathcal{H}\left\{h_{n}(x)\right\} * f(x)=E_{n}(x) e^{i \phi_{n}(x)},
$$

where $h$ is given family of band-pass filters and $n$ indexes a particular scale. $h$ and $\mathcal{H}\{h\}$ form a quadrature pair. Quadrature filters are an ubiquitous topic in computer vision and an extensive review can be found in [1. In this paper, we have chosen a log-Gabor filter as our quadrature pair. The log-Gabor filter pair is defined in the Fourier domain by $G(\omega)=e^{-\frac{\log ^{2}\left(\frac{\omega}{\omega_{0}}\right)}{2 \log ^{2}\left(\frac{k}{\omega_{0}}\right)}}$, where $\omega_{0}$ is the center frequency of the filter and $\frac{\kappa}{\omega_{0}}$ is a constant factor that defines the filter bandwidth. Then $h$ and $\mathcal{H}\{h\}$ are obtained as the real and imaginary part of the inverse Fourier transform of $G$ respectively.

Phase congruency, $\Psi$, is a normalized measure of phase variance across scales. In terms of the local phase, phase congruency is defined as

$$
\Psi(x)=\frac{\sum_{n=1}^{N} E_{n}(x) \cos \left(\phi_{n}(x)-\bar{\phi}_{n}(x)\right)}{\sum_{n} E_{n}(x)},
$$

where $\bar{\phi}_{n}(x)$ is the average local phase and $N$ is the number of bands used in the analysis. Venkatesh and Owens 9] have shown that phase congruency is proportional to local energy and it can be calculated from the analytic signal components as

$$
\Psi(x)=\frac{\sqrt{\left(\sum_{n=1}^{N} \operatorname{Re}\left\{\mathbf{f}_{A_{n}}\right\}\right)^{2}+\left(\sum_{n=1}^{N} \operatorname{Im}\left\{\mathbf{f}_{A_{n}}\right)^{2}\right\}}}{\sum_{n=1}^{N}\left\|\mathbf{f}_{A_{n}}\right\|}
$$

Phase congruency is a smooth function with local maxima in locations where the local phase is consistent across scales. For our application, it is very interesting to be able to distinguish between locations where the local maxima in phase congruency is due to the inner wall edge or the outer wall edge. This distinction can be easily achieved by using the feature encoding properties of the local phase. In this paper, we introduce a new measure for phase congruency: feature dependent phase congruency, $\Psi_{\theta}$, given by

$$
\Psi_{\theta}(x)=\Psi(x) \max (\cos (\bar{\phi}(x)-\theta), 0),
$$

where $\theta$ depends on the feature type we want to tune the phase congruency measure. For a dark-to-bright edge $\theta=\pi / 2$ and for a bright-to-dark edge $\theta=\frac{3 \pi}{4}$. Therefore, the inner/outer wall is given by the location that maximizes $\Psi_{\pi / 2}$ and $\Psi_{3 \pi / 4}$ respectively,

$$
a_{i}=\arg \max \Phi_{\pi / 2}(x), \quad a_{o}=\arg \max \Phi_{3 \pi / 4}(x) .
$$

The performance of the proposed phase congruency measure can be seen in Figure 2 $\mathrm{b}$-c in comparison with the traditional measure for phase congruency. The 
traditional measure does not provide feature selectivity and phase congruency has a flat maxima region across the whole airway wall. However, the proposed measurement adds localization based on the corresponding feature.

\subsection{Bronze Standard}

From the experimental results presented in Section 3.1, we proposed a bronze standard for wall thickness location based on the intersecting point between different reconstruction kernel acquisitions for the same CT Radon space acquisition. We define the location of the wall as the median intersection point for all possible pair kernel combinations. The median operator is used because it is a robust estimator.

\section{Materials}

The evaluation and validation of the proposed method has been carried out by means of a computer-based airway simulator, a CT phantom of airways and a CT scan of a human lung.

Characterizing the method robustness against noise is an important metric to assess the applicability of the method to clinical-quality CTs obtained with low dose protocols. Low dose protocols can be modeled by an increment of the amount of additive Gaussian noise in the Radon space. Our simulator works as follows: an ideal airway is transformed to the Radon space. In the Radon space, independent Gaussian noise with power $\sigma_{n}$ is added to the data. The noise-corrupted data is smoothed and downsampled to simulate different scanner reconstruction kernels and scanner resolutions respectively. The data is transformed back to the spatial domain by means of the backprojection transform. We use this simulator to generate different airway models. The airways were sampled in a $0.6 \mathrm{~mm}$ image.

A CT airway phantom was constructed using Nylon66 tubing inserted into polystyrene to simulate lung parenchyma surrounding the airways. Non-overlapping, $0.6 \mathrm{~mm}$ collimation images, $40 \mathrm{~cm}$ FOV, were acquired using a Siemens Sensation 64 CT scanner and reconstructed with a series of 9 different kernels provided by the manufacturer. Two tubes with wall thickness $a_{0}-a_{i}=0.9 \mathrm{~mm}$ and $a_{0}-a_{i}=1.05 \mathrm{~mm}$, as measured by a caliper, were studied.

Finally, a CT scan of a human lung was used to evaluate the behavior of our method on real data. Images were acquired using the Siemens Sensation 64 CT scanner, non-overlapping isotropic voxels of $0.6 \mathrm{~mm}$ in dimension. Reconstructions of the raw data were obtained using the same 9 kernels utilized for the phantom investigation. Two representative airways were picked by an expert.

\section{Results}

All the results for phase congruency were obtained using a log-Gabor filter bank. 10 bands were used for the frequency decomposition. The filter bandwidth was 


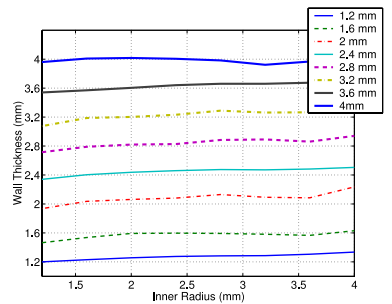

(a)

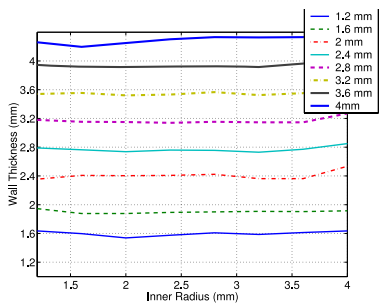

(b)

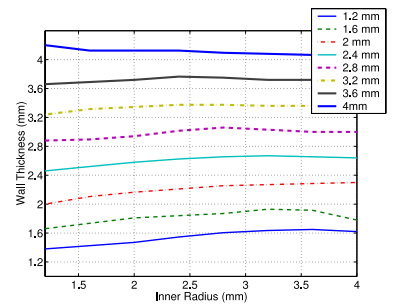

(c)

Fig. 3. Wall thickness analysis using the computerized phantom for airways. Airways with different radius and wall thickness sizes have been simulated. (a) Validation of the bronze standard for airway wall location. (b) FWHM and (c) Phase congruency wall thickness estimation.

given by $\frac{\kappa}{\omega_{0}}=0.85$ and the central frequency for the $i t h$ band was given by $\omega_{0}^{i}=\frac{2}{\lambda_{i}}$ with $\lambda_{i}=\alpha^{i} \lambda_{\min }$, where $\lambda_{\min }$ is the minimum wavelength of the filter bank. In our case we chose $\alpha=1.3$ and $\lambda_{\text {min }}=4$ pixels. The election of the parameters was based on experimental results based on the scale of typical airways.

\subsection{Computer Phantom}

The validity of the bronze standard was tested simulating different airway sizes with different smoothing kernel parameters. Figure 3 a shows the results obtained for different airway thicknesses estimated at different inner radius level. Ideally, the plot should be a straight line at the wall thickness level corresponding to the tested airway. The bronze standard yields an accurate measure, up to discretization error, with no bias in the measurement. The error in the estimation is slightly bigger for the thinnest airways $(1.2 \mathrm{~mm}$ and $1.6 \mathrm{~mm})$ given that we are close to the sampling limitation imposed by the Nyquist theorem. Similar plots are shown in Figure $3 \mathrm{~b}$ and $3 \mathrm{c}$ for the wall thickness estimated by FWHM and phase congruency respectively. Although there is a bias, as reported by [7], in the estimation of the wall thickness, phase congruency estimations are closer to the expected values. Therefore, phase congruency is more accurate than FWHM across the whole range of airway sizes.

Finally, we studied the robustness of FWHM and phase congruency for wall estimation against noise. Figure 4 a shows the wall thickness estimation for an airway with inner radius of $4 \mathrm{~mm}$ and wall thickness of $2 \mathrm{~mm}$ at different SNR levels. For each noise level, 80 realizations were performed. The plot shows the mean wall thickness and the standard deviation is shows as vertical rectangles. FWHM has a great sensitivity due to noise given by a larger slope when the SNR decreases. There is a critical point at $10 \mathrm{~dB}$ in both methods at which the slope increases; this point imposes a limit to the amount of noise allowed for accurate wall detection. Although the standard deviation is similar for both methods, it is slightly smaller for phase congruency. 


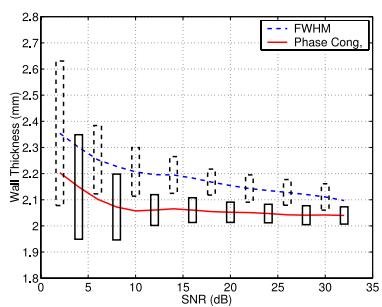

(a)

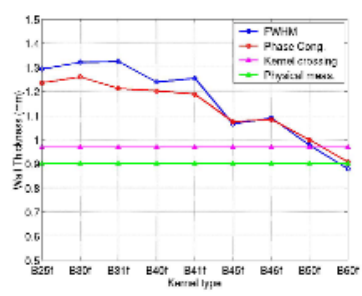

(b)

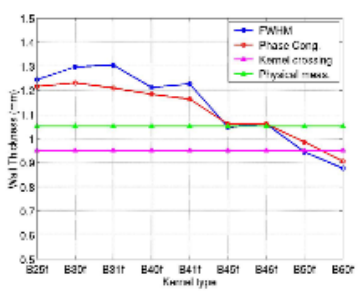

(c)

Fig. 4. (a) Noise analysis for FWHM and Phase Congruency for an $2 \mathrm{~mm}$ thick airway using our airway simulator. (b)-(c) Wall thickness measurements for the airway phantom at different reconstruction kernels: (b) Tube 1 (0.9 $\mathrm{mm}$ wall thickness) and (c) Tube 2 (1.05 $\mathrm{mm}$ wall thickness).

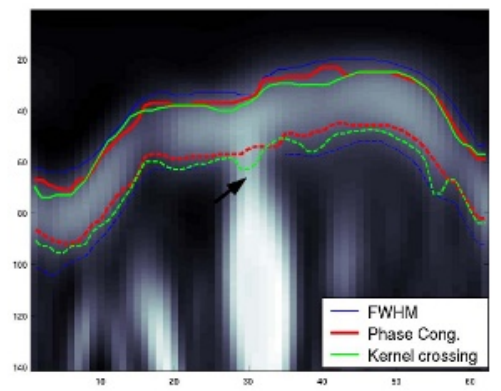

(a)

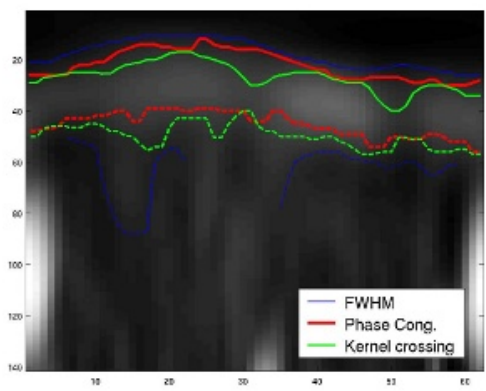

(b)

Fig. 5. Wall Estimation for a real CT scan for two different size airways. A continuous line corresponds to the inner boundary and a dash line to the outer boundary. FWHM has difficulties recovering the outer boundary when the wall has not enough contrast or vessels are in close contact with the wall.

\subsection{Airway Phantom}

The results for the airway phantom are shown in Figure $4 \mathrm{~b}-\mathrm{c}$. We can see how accurate the bronze standard predicts the wall thickness of the tube. This corroborates the use of the bronze standard for the validation of methods to detect thin structures in CT, like the airway walls. FWHM and phase congruency yield an overestimation of the wall thickness for smooth kernels as predicted by our simulation. Better results can be though achieved using phase congruency.

\subsection{Lung CT}

The detected wall for two unfolded airways are shown in Figure 5, Although we have datasets for each different reconstruction kernel, only the B46f kernel was employed to estimate the wall using FWHM and phase congruency. Unlike the 
previous results, real data has a greater complexity due to surrounding structures that complicate the detection of the outer wall. As it can be easily seen in Figure 5a, FWHM breaks when trying to detect the outer wall boundary when it is abutting a blood vessel. This is in part because these areas do not fit the model that FWHM was developed for. When looking at airways where the contrast between wall and parenchyma is very subtle, phase congruency shows its superiority with respect to FWHM, as shown in Figure 5b. FWHM is not able to yield any reliable measure for the outer boundary of the wall. Comparing against the bronze standard, we can say that phase congruency gives an accurate estimation of the wall boundaries.

\section{Conclusions}

We have proposed a new method for airway wall detection based on the principle of phase congruency. The method that we propose is based on a new definition for phase congruency that makes the traditional phase congruency phase dependent. We have seen that this new phase dependent phase congruency yields a sharper response with clear maxima in the locations of edges and lines.

The measurement of the wall thickness has been traditionally based on the FWHM method. We have corroborated the results presented in [7] that quantifies the overestimation on the wall measurement when using FWHM. Our method, while still sensitive to this overestimation, gives a better result than FWHM, as shown by synthetic and real data examples It would be very interesting to study the sources of this overestimation in the phase congruency method by evaluating the analytic expressions of the filters responses.

Phase congruency appears to be suitable for the wall estimation in low dose CT scans based on the results for different noise levels. In part, the analysis by bands, that phase congruency is based on, explains the capability to handle noise better than FWHM. Another important characteristic of our method is the lower sensitivity to the reconstruction kernel selection. This is crucial when applying the method to retrospective data and data acquired across multiple sites. Finally, an important contribution of this paper has been the proposal of a bronze standard for the accurate detection of the airway wall based on the isoline for the intersection across multiple reconstruction kernels.

\section{References}

1. D. Boukerroui, J. Alison Noble, and M. Brady. On the choice of band-pass quadrature filters. Journal of Mathematical Imaging and Vision, 21:53-80, 2004.

2. S. Hu, E. A. Hoffman, and J. M. Reinhardt. Automatic lung segmentation for accurate quantitation of voumetric x-ray ct images. IEEE Trans. Med. Imaging, 20:490-498, 2001.

3. Gregoire Malandain, Gilles Bertrand, and Nicholas Ayache. Topological segmentation of discrete surfaces. Int. Journal of Computer Vision, 10(2):183-197, 1993.

4. M. C. Morrone and R. A. Owens. Feature detection from local energy. Pattern Recognition Letters, 6:303-313, 1987. 
5. Y. Nakano and S. Muro et al. Computed tomographic measurements of airway dimensions and emphysema in smokers. Am J REspir Crit Care Med, 162:11021108, 2000.

6. Eric Pichon, Allen Tannenbaum, and Ron Kikinis. A statistically based flow for image segmentation. Medical Image Analysis, 8(3):267-274, 2004.

7. Joseph M. Reinhardt, Neil D. D'Souza, and Eric A. Hoffman. Accurate measurement of intra-thoracic airways. IEEE Trans. Medical Imaging, 16(6):820-827, Dec. 1997.

8. Osama Saba, Eric A. Hoffman, and Joseph M. Reinhardt. Maximizing quantitative accuracy of lung airway lumen and wall measures obtained from X-ray CT imaging. J. Applied Physiology, 95:1063-1095, 2003.

9. S. Venkatesh and R. Owens. On the classification of image features. Pattern Recognition Letters, 11:339-349, 1990. 